\title{
BMJ Open Sputum PGP is reduced by azithromycin treatment in patients with COPD and correlates with exacerbations
}

\author{
Philip J O’Reilly, ${ }^{1}$ Patricia L Jackson, ${ }^{1} \mathrm{~J}$ Michael Wells, ${ }^{1}$ Mark T Dransfield, ${ }^{1}$ \\ Paul D Scanlon, ${ }^{2} \mathrm{~J}$ Edwin Blalock ${ }^{1}$
}

To cite: O'Reilly PJ, Jackson PL, Wells JM, et al. Sputum PGP is reduced by azithromycin treatment in patients with COPD and correlates with exacerbations. BMJ Open 2013;3:e004140. doi:10.1136/bmjopen-2013004140

- Prepublication history for this paper is available online. To view these files please visit the journal online (http://dx.doi.org/10.1136/ bmjopen-2013-004140).

Received 3 October 2013 Revised 19 November 2013 Accepted 20 November 2013

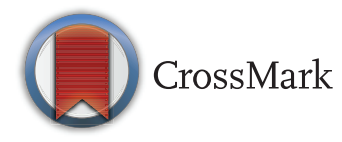

For numbered affiliations see end of article.

Correspondence to Dr Philip J O'Reilly; poreilly@uab.edu

\section{ABSTRACT}

Rationale: Proline-glycine-proline (PGP), a neutrophil chemoattractant derived from the enzymatic breakdown of collagen, is elevated in sputum of patients with chronic obstructive pulmonary disease (COPD) and may contribute to disease progression. Whether sputum levels of PGP respond to therapy for COPD or predict outcomes is unknown.

Objectives: We conducted a study ancillary to a multicenter trial of the efficacy of azithromycin treatment for 1 year in preventing COPD exacerbations to test whether sputum levels of PGP were altered by treatment or associated with exacerbation frequency.

Methods: We collected remnant sputa from trial participants and assayed them in a blinded fashion for PGP, myeloperoxidase and matrix metalloproteinase (MMP)-9 and for the ability to generate PGP from collagen ex vivo. Once the parent trial was unblinded, the results were correlated with use of azithromycin or placebo and exacerbations in participants.

Results: Azithromycin treatment significantly reduced sputum levels of PGP and myeloperoxidase in patients with COPD, particularly with increased duration of therapy. We found no difference in sputum MMP-9 or PGP generation between participants taking azithromycin or placebo. Sputum PGP levels were highest around the time of an exacerbation and declined with successful treatment.

Conclusions: These data support a role for PGP in the airway and parenchymal neutrophilic inflammation that drives COPD progression and exacerbations, and provide new information on the anti-inflammatory properties of macrolides. PGP may have potential as a target for novel anti-inflammatory therapies in COPD and as a biomarker for clinical trials.

\section{INTRODUCTION}

Proline-glycine-proline (PGP) is a tripeptide generated from the breakdown of extracellular matrix collagen and is specifically chemotactic for neutrophils in vitro and in vivo. ${ }^{1}$ PGP exerts its chemotactic effect through sequence homology with a key motif found in ELR $^{+}$CXC chemokines and binds to their

\section{Strengths and limitations of this study}

- This research was conducted ancillary to a multicenter, prospective, parallel group, placebocontrolled, double-blind study of the efficacy of azithromycin in the chronic, outpatient management of chronic obstructive pulmonary disease (COPD).

- We demonstrate that chronic treatment with azithromycin reduces levels of proline-glycineproline (PGP), a collagen-derived neutrophil chemoattractant, in sputum of patients with COPD.

- This provides new information on the antiinflammatory properties of macrolide antibiotics and supports a role for PGP in COPD pathogenesis and as a biomarker in clinical trials.

- The number of participants included in this study is small as sputum samples were available only from a minority of participants in the parent trial.

receptors, CXCR1 and CXCR2. ${ }^{1}$ PGP is generated from native collagen by the action of neutrophil-derived matrix metalloproteinases (MMPs) 8 and 9, which cleave collagen into oligopeptides, followed by a secondary cleavage by prolyl endopeptidase, ${ }^{2}$ a neuronal enzyme implicated in hypertension and neuropeptide processing. Prolyl endopeptidase is also present in neutrophils where it likely participates in the generation of PGP $^{3}$ PGP is broken down in the lung by the aminopeptidase activity of leukotriene $\mathrm{A}_{4}$ hydrolase $\left(\mathrm{LTA}_{4} \mathrm{H}\right)$, an enzyme hitherto recognised only as proinflammatory through generation of leukotriene $\mathrm{B}_{4}{ }^{4}$ Consequently, acute PGP-driven neutrophilic inflammation may be terminated by $\mathrm{LTA}_{4} \mathrm{H}$. Cigarette smoke can selectively inactivate the aminopeptidase, but not the hydrolase, activity of $\mathrm{LTA}_{4} \mathrm{H}$, thus allowing accumulation of PGP, while promoting neutrophilic inflammation. ${ }^{4}$

We have previously demonstrated that PGP is a potential sputum and serum biomarker 
in chronic obstructive pulmonary disease (COPD), cystic fibrosis (CF) and chronic lung transplant rejection and contributes to neutrophilic airway inflammation in these diseases. $^{2}{ }^{5}{ }^{6}$ Sputa from patients with $\mathrm{CF}$ and COPD generate PGP from collagen ex vivo. ${ }^{25}$ Levels of PGP in $\mathrm{CF}$ sputum are higher during exacerbations and decline with successful treatment and resolution. ${ }^{2}$ Whether PGP levels are modifiable by outpatient therapy of lung diseases or whether such changes can positively impact clinical outcomes has not been tested. This is important, as no biomarker has yet been identified that can reliably aid in diagnosis and phenotyping of COPD or serve as a surrogate endpoint for clinical trials. Identifying such biomarkers is a key priority. ${ }^{78}$

Existing pharmacotherapies do not alter the neutrophilic airway and parenchymal inflammation that are at the heart of disease progression in COPD and consequently do not prevent loss of lung function. Accordingly, there is a need for new therapies. One candidate is azithromycin, a macrolide antibiotic with anti-inflammatory properties. Azithromycin and other macrolides have been proven beneficial in chronic, neutrophilic airway diseases, such as bronchiectasis, diffuse panbronchiolitis and CF. ${ }^{9}{ }^{10}$ These effects are believed to be due, in part, to an as yet unexplained anti-inflammatory activity and not just suppression of bacterial growth. ${ }^{11}$

The National Heart Lung and Blood Institute (NHLBI) recently sponsored a multicenter trial of azithromycin in stable outpatients with COPD (the Macrolide trial). ${ }^{12}$ As macrolides have been demonstrated to reduce MMP activity in lung diseases, including COPD, ${ }^{11} 1314$ we hypothesised that beneficial effects of azithromycin might be mediated through reducing PGP. Accordingly, we conducted a study ancillary to the Macrolide trial in which sputum collected from participants for microbial analysis was analysed for PGP and the ability to generate PGP from collagen. Once the code for the parent trial was broken, we correlated PGP levels with use of azithromycin or placebo and clinical response. The results are herein reported.

\section{METHODS}

Patient recruitment

This research was carried out as an ancillary study to the Macrolide trial of the NHLBI COPD Clinical Research Network (CRN). This was a prospective, parallel group, placebo-controlled, double-blind study of the efficacy of azithromycin in the chronic, outpatient management of COPD with a primary outcome of time to first exacerbation. ${ }^{12}$ Patients with COPD, selected to be at high risk of acute exacerbation, were randomised to receive either azithromycin $250 \mathrm{mg}$ daily or placebo for 12 months. Nasopharyngeal swabs were obtained from participants at various points during the trial (at randomisation, after 1 , $3,6,9$ and 12 months of treatment and 1 month after completion) to screen for colonisation by macrolide-resistant pathogens. In a limited number of participants, sputum samples were also obtained for microbial analysis. For the current study, we obtained remnant sputum from these participants for measurement of biomarkers.

\section{Measurement of PGP, myeloperoxidase and MMP-9 in sputum}

Sputum was diluted 1:1 with normal saline, filtered through a $10 \mathrm{kDa}$ filter, washed with $40 \mu \mathrm{L}$ of $1 \mathrm{~N} \mathrm{HCl}$ and assayed for PGP by electrospray ionisation-liquid chromatography-mass spectrometry/mass spectrometry (ESI-LC-MS/MS) as previously described. ${ }^{2} 5$ The detection limit was $10 \mathrm{pg} / \mathrm{mL}$.

Myeloperoxidase was measured in sputum using a commercially available activity assay (Calbiochem, San Diego, California, USA). Samples and standards were added to wells coated with a polyclonal antibody to human myeloperoxidase and incubated for $2 \mathrm{~h}$. The detection reagent (tetramethylbenzidine and hydrogen peroxidase) was added, and after $1 \mathrm{~h}$ the absorbance was read at $450 \mathrm{~nm}$. Activity was converted to $\mathrm{ng} / \mathrm{mL}$ active myeloperoxidase using a standard curve.

The concentration of total and pro-MMP-9 was determined in sputum using commercially available ELISA kits (R\&D Systems, Minneapolis, Minnesota, USA). Samples and standards were added to wells coated with a monoclonal antibody to MMP-9 and incubated for $2 \mathrm{~h}$ at room temperature. A fluorogenic substrate (Fluor-Pro-Leu-Gly-Leu-Ala-Arg- $\mathrm{NH}_{2}$ ) was added and the plate was incubated for $18 \mathrm{~h}$ at $37^{\circ} \mathrm{C}$. Activity was quantified using a spectrophotometer with excitation and emission wavelengths of 320 and $450 \mathrm{~nm}$, respectively, and converted to $\mathrm{ng} / \mathrm{mL}$ active MMP-9 using a standard curve.

\section{Generation of PGP ex vivo}

One hundred microlitre of sputum was incubated with extensively dialysed (against $\mathrm{dH}_{2} \mathrm{O}$ with $\mathrm{pH}$ adjusted to 4.5 by addition of glacial acetic acid) intact type I or II collagen $(10 \mu \mathrm{L}, 1 \mathrm{mg} / \mathrm{mL}$, Sigma-Aldrich, St Louis, Missouri, USA) for $24 \mathrm{~h}$ at $37^{\circ} \mathrm{C}$ and $5 \% \mathrm{CO}_{2}$. The samples were filtered through a $10 \mathrm{kDa}$ filter, washed with $40 \mu \mathrm{L}$ of $1 \mathrm{~N} \mathrm{HCl}$ and analysed using ESI-LC-MS/ MS for levels of PGP. Amounts of PGP generated by each sample were determined by comparison with PGP generated by sputum incubated without collagen.

\section{Data analysis}

Sputum samples were identified as coming from participants taking azithromycin or placebo once the Macrolide trial was completed and unblinded. The number of exacerbations for each participant and the date of onset of symptoms for each exacerbation were recorded. Data are presented as means \pm SEM. Sputum biomarker levels and activities were compared between groups using Students $\mathrm{t}$ test. All $\mathrm{p}$ values $<0.05$ were considered significant. 


\section{RESULTS}

Azithromycin reduces sputum PGP and myeloperoxidase compared with placebo in a time-dependent fashion

We obtained a total of 46 sputum samples from patients with COPD recruited to the Macrolide trial and assayed them in a blinded fashion for levels of PGP. Unblinding of the parent trial revealed that there were 18 sputum samples from 13 placebo-treated participants and 14 sputum samples from 8 azithromycin-treated participants collected at months 1 through 12 of treatment. Clinical characteristics of our study participants were similar to those of the parent trial (age $=67.6 \pm 8.4$ years, $30 \%$ female, postbronchodilator $\mathrm{FEV}_{1}=1.36 \pm 0.47 \mathrm{~L}$, mean $\pm \mathrm{SD}$ ).

Sputum PGP levels were significantly higher in participants taking placebo compared with those taking azithromycin during the trial $(5.15 \pm 1.54$ vs $2.27 \pm 0.87 \mathrm{ng} / \mathrm{mL}$, $\mathrm{p}<0.05$; figure 1$)$. The difference in sputum PGP was greatest at months 9 and 12, indicating a cumulative effect of azithromycin treatment (figure 2). Given the limited data set, effects of azithromycin treatment on PGP levels are reported for groups. However, there was a progressive decline in sputum PGP with duration of treatment in five of six azithromycin-treated participants whose sputum was assayed at more than one time point.

The difference in sputum myeloperoxidase activity between participants taking placebo or azithromycin over the 12 months of the trial was not statistically significant ( $3391 \pm 353$ vs $2884 \pm 349 \mathrm{ng} / \mathrm{mL}, \mathrm{p}=0.16)$. However, sputum myeloperoxidase was lower in participants taking azithromycin from months 6 through $12(3321 \pm 430$ vs 2398 $\pm 290 \mathrm{ng} / \mathrm{mL}, \mathrm{p}<0.05$ ), indicating a cumulative effect of azithromycin treatment on neutrophilic airway inflammation.

There was no difference between participants taking placebo or azithromycin in sputum MMP-9 activity $(11.18 \pm 3.98$ vs $8.39 \pm 2.42 \mathrm{ng} / \mathrm{mL}$, NS) or PGP generation from collagen ex vivo $(3.28 \pm 2.25$ vs $2.94 \pm 1.73 \mathrm{ng} / \mathrm{mL}$, NS) during the trial.

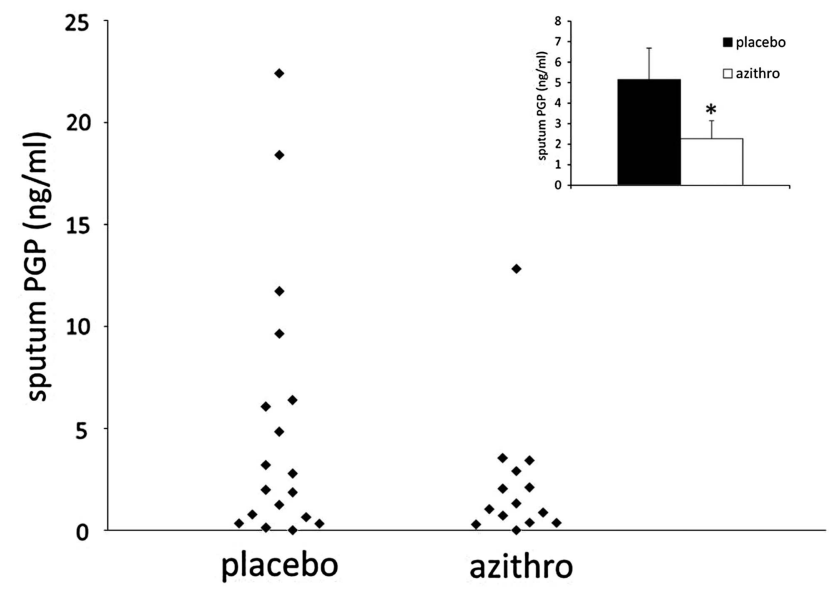

Figure 1 Azithromycin treatment decreases proline-glycineproline (PGP) levels in sputum of patients with chronic obstructive pulmonary disease compared with placebo. Inset: values represent the mean \pm SEM PGP concentrations for sputa ( ${ }^{*} \mathrm{p}<0.05$ vs placebo).

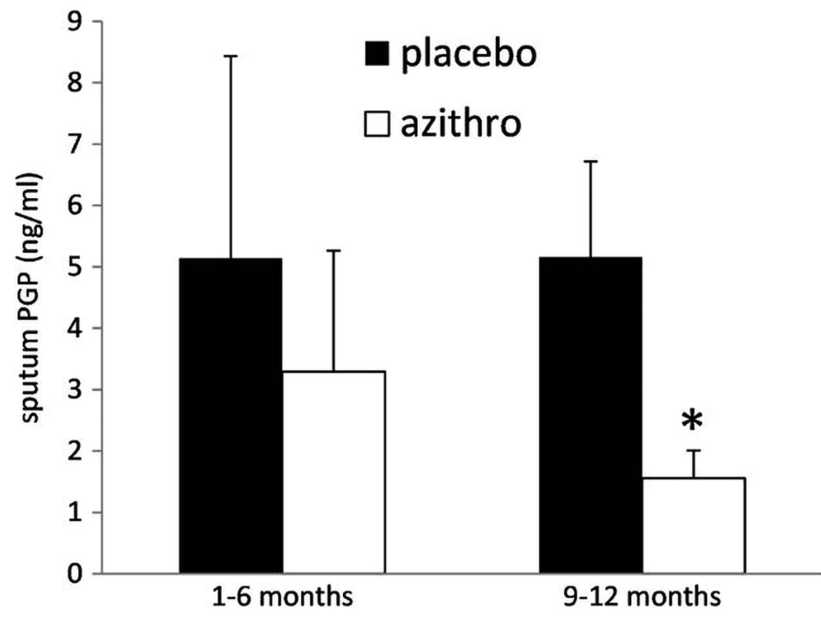

Figure 2 Sputum proline-glycine-proline (PGP) levels decline with increased duration of azithromycin therapy. PGP levels were significantly higher in participants treated with placebo compared with azithromycin at months 9 and 12 of therapy $\left({ }^{*} p<0.02\right)$ but not at months 1 through $6(p=0.32)$.

Fall in sputum PGP is associated with reduced exacerbation frequency in patients with COPD

In the Macrolide trial, treatment with azithromycin significantly reduced exacerbation frequency in high-risk patients with COPD compared with placebo (1.48 vs 1.83 exacerbations per patient year, $\mathrm{p}=0.01$ ) and increased the time to first exacerbation. ${ }^{12}$ We saw a similar reduction in exacerbation frequency in participants taking azithromycin in our ancillary study although this was not significant due to the small size of our study group $(0.91 \pm 0.37$ vs $1.93 \pm 0.62$ exacerbations per patient year, $\mathrm{p}=0.08$ ).

\section{Sputum PGP rises during COPD exacerbations and declines with resolution}

We obtained the dates of exacerbations experienced by our participants during the Macrolide trial, including placebo and azithromycin-treated groups, and correlated sputum PGP with time in days from the closest exacerbation. This resulted in an interesting pattern that suggested that PGP levels precipitously rise in advance of an exacerbation and rapidly decline thereafter (figure 3). Indeed, sputum PGP was higher around the time of an exacerbation (18 \pm 4 days before or after, $12.8 \pm 4.53 \mathrm{ng}$ $\mathrm{PGP} / \mathrm{mL} ; \mathrm{n}=9)$ than long before ( $103 \pm 37$ days, 1.10 $\pm 0.411 \mathrm{ng}$ PGP $/ \mathrm{mL}, \mathrm{n}=6$; $\mathrm{p}<0.02$ ) or after an exacerbation $(141 \pm 11$ days, $2.15 \pm 0.80 \mathrm{ng} \mathrm{PGP} / \mathrm{mL}, \mathrm{n}=11, \mathrm{p}<0.02$; figure 3 inset). There was a trend towards higher sputum PGP after an exacerbation than before $(\mathrm{p}=0.133)$.

\section{DISCUSSION}

PGP is a neutrophil chemoattractant in vitro and in vivo and contributes to airway inflammation in chronic lung diseases with a prominent neutrophil component including COPD and $\mathrm{CF}^{2}{ }^{5}{ }^{6}$ Chronic instillation of PGP into mouse lungs results in emphysema and right ventricular 
Figure 3 Sputum prolineglycine-proline (PGP) levels are highest around the time of a chronic obstructive pulmonary disease exacerbation. Values represent PGP levels at various days relative to the onset of an exacerbation (time 0). Inset: values represent the mean \pm SEM PGP concentrations for sputa collected $103 \pm 37$ days before an exacerbation (pre), $18 \pm 4$ days before or after an exacerbation (peri) or $141 \pm 11$ days after an exacerbation (post). ${ }^{*} \mathrm{p}<0.02$ (pre vs peri), ${ }^{\#} p<0.02$ (post vs peri).

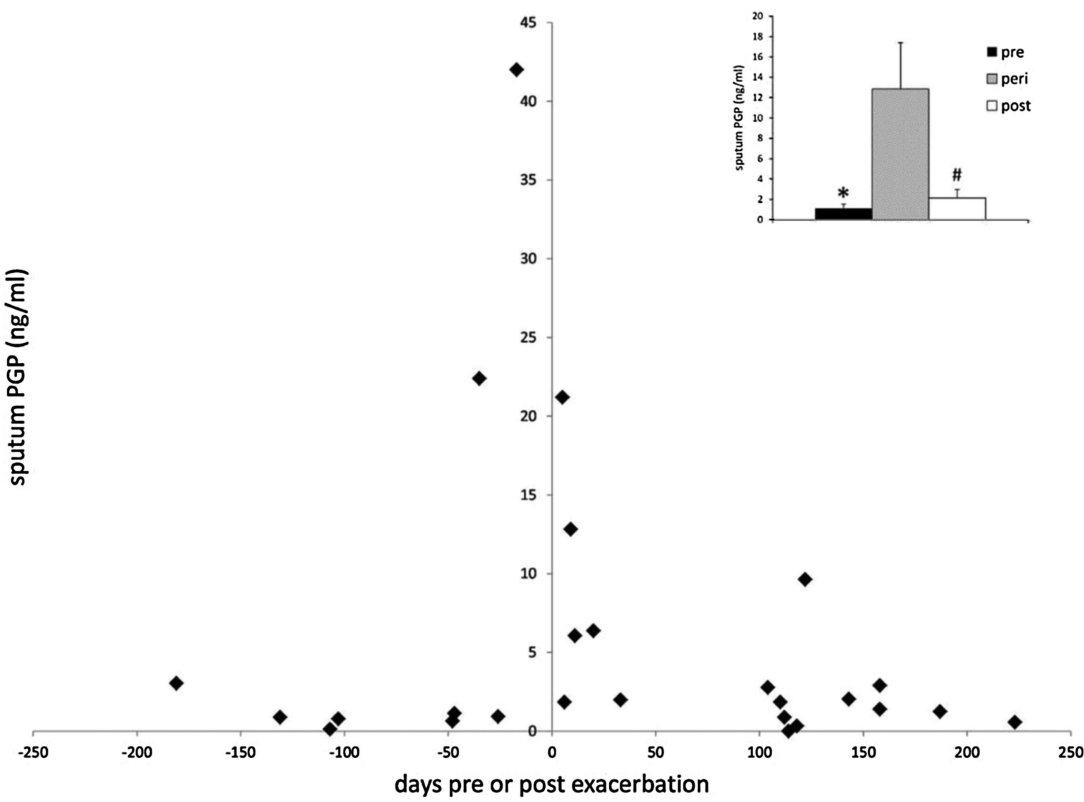

hypertrophy ${ }^{1}$ and inhibition of PGP by a complementary peptide (arginine-threonine-arginine) reduces acute cigarette smoke-induced neutrophil influx into mouse lungs and prevents emphysema and right ventricular hypertrophy in a mouse model of COPD. ${ }^{15}{ }^{16}$ However, direct evidence implicating PGP in COPD pathogenesis in humans is lacking. In the limited number of sputum samples available to us due to the design of the Macrolide trial, we demonstrate that PGP is reduced in the airways of patients with COPD taking azithromycin, particularly with longer duration of therapy and is accompanied by reduced neutrophil burden, measured as sputum myeloperoxidase. Reduction in sputum PGP is accompanied by a positive clinical response to azithromycin (reduced exacerbation frequency). Our subgroup had a similar therapeutic response to that seen in the parent trial, suggesting that our findings could be generalised to the entire Macrolide group.

Although seen with only a few specimens, sputum PGP was strikingly elevated as long as 35 days before the onset of symptoms of an acute exacerbation of COPD and briefly afterwards. It declined sharply with successful treatment and resolution (figure 3). Seemungal et $a l^{17}$ described a 7-days prodrome where respiratory symptoms worsened before the onset of an exacerbation but failed to predict exacerbations with spirometry. Our data suggest that airway inflammation may worsen weeks in advance of this and that PGP may predict the onset of an exacerbation long enough before symptoms appear to permit early intervention. Although many biomarkers of COPD have been described, to our knowledge, none has been shown to herald exacerbations. ${ }^{18}$ The persistence of elevated sputum PGP long after exacerbations in some individuals may contribute to continuing neutrophilic airway inflammation and promote the 'frequent exacerbator' phenotype. ${ }^{19-21}$
We cannot determine from our data whether the reduction in exacerbations due to azithromycin was secondary to reduced sputum PGP or whether the reduction in sputum PGP was due to fewer exacerbations in azithromycin-treated participants. Answering this question definitively would require a larger, longitudinal study of PGP in patients with COPD. Nevertheless, these data support the idea that PGP plays a significant role in COPD pathogenesis, particularly exacerbations, and may mycin's anti-inflammatory properties. PGP predicted a positive response to azithromycin within 6 months of starting therapy and was superior in this respect to other biomarkers studied as part of the Macrolide trial. ${ }^{22}$

We are uncertain as to the mechanism of PGP reduction by azithromycin. Macrolide antibiotics have been reported to have numerous anti-inflammatory properties, including inhibiting MMP activity. ${ }^{11} 1314$ As MMP-8 and MMP-9 are essential to extracellular PGP generation from collagen by neutrophils, ${ }^{2}$ this would provide an attractive potential mechanism. However, we could find no difference in sputum MMP-9 activity between treatment groups. We did not examine other MMPs which we have previously implicated in PGP generation, such as MMP-1 and MMP-8. ${ }^{2} 5$ We measured PGP generation ex vivo from collagen by sputum but could find no difference between treatment groups. Subsequent to the current study, we have identified a neutrophil-derived enzyme, $\mathrm{LTA}_{4} \mathrm{H}$, which generates leukotriene $\mathrm{B}_{4}$ and rapidly degrades PGP. $^{4}$ We have recently found that $\mathrm{LTA}_{4} \mathrm{H}$ is markedly elevated in COPD sputum compared to controls. ${ }^{23}$ Breakdown of PGP by $\mathrm{LTA}_{4} \mathrm{H}$ in our sputum samples could have confounded the PGP generation assay. As PGP is derived from neutrophil activity, reduced sputum PGP might reflect reduced recruitment of neutrophils to the airways due to other effects of provide important clues as to the mechanism of azithro- 
azithromycin. The decline in sputum PGP was greater than the decline in myeloperoxidase, suggesting that PGP may have other effects in COPD aside from recruiting neutrophils. Addressing these questions was outside the scope of this study.

In conclusion, the results of our study support the idea that PGP plays an important role in COPD pathogenesis and provide clues as to the anti-inflammatory mechanism of azithromycin. PGP shows promise as a predictor of acute exacerbations and may serve as a useful biomarker for response to therapy in future studies of COPD and other chronic, neutrophilic lung diseases.

Author affiliations

${ }^{1}$ Division of Pulmonary, Allergy and Critical Care Medicine, Lung Health Center, University of Alabama at Birmingham, Birmingham, Alabama, USA ${ }^{2}$ Department of Pulmonary and Critical Care Medicine, Mayo Clinic, Rochester, Minnesota, USA

Acknowledgements The authors would like to thank the National Heart Lung and Blood Institute chronic obstructive pulmonary disease clinical research network for their assistance in providing sputum samples and clinical data for this study.

Contributors PJO designed the study, acquired and interpreted the data and drafted the manuscript. PLJ and JMW acquired and interpreted the data. MTD and PDS provided sputum samples. JEB designed the study and supervised the project. All authors contributed to revision and final approval of the manuscript.

Funding Funding for this research came from grants HL090999, HL110950, HL077783 (to JEB) and HL092296 (to PJO) from the National Heart and Blood Institute of the National Institutes of Health.

Competing interests None.

Ethics approval Ethics approval for this study was provided by the Institutional Review Board at the University of Alabama at Birmingham. Approval for this study was also provided by the Steering Committee of the NHLBI COPD Clinical Research Network which conducted the original trial. Registration for the original trial is at clinicaltrials.gov, identifier: NCT00325897.

Provenance and peer review Not commissioned; externally peer reviewed.

Data sharing statement No additional data are available.

Open Access This is an Open Access article distributed in accordance with the Creative Commons Attribution Non Commercial (CC BY-NC 3.0) license, which permits others to distribute, remix, adapt, build upon this work noncommercially, and license their derivative works on different terms, provided the original work is properly cited and the use is non-commercial. See: http:// creativecommons.org/licenses/by-nc/3.0/

\section{REFERENCES}

1. Weathington NM, van Houwelingen $\mathrm{AH}$, Noerager BD, et al. A novel peptide CXCR ligand derived from extracellular matrix degradation during airway inflammation. Nat Med 2006;12:317-23.
2. Gaggar A, Jackson PL, Noerager BD, et al. A novel proteolytic cascade generates an extracellular matrix-derived chemoattractant in chronic neutrophilic inflammation. J Immunol 2008;180:5662-9.

3. O'Reilly PJ, Hardison MT, Jackson PL, et al. Neutrophils contain prolyl endopeptidase and generate the chemotactic peptide, PGP, from collagen. J Neuroimmunol 2009;217:51-4.

4. Snelgrove RJ, Jackson PL, Hardison MT, et al. A critical role for LTA4H in limiting chronic pulmonary neutrophilic inflammation. Science 2010;330:90-4.

5. O'Reilly P, Jackson PL, Noerager B, et al. N-alpha-PGP and PGP, potential biomarkers and therapeutic targets for COPD. Respir Res 2009;10:38.

6. Hardison MT, Galin FS, Calderon CE, et al. The presence of a matrix-derived neutrophil chemoattractant in bronchiolitis obliterans syndrome after lung transplantation. J Immunol 2009;182:4423-31

7. Croxton TL, Weinmann GG, Senior RM, et al. Clinical research in chronic obstructive pulmonary disease: needs and opportunities. Am J Respir Crit Care Med 2003;167:1142-9.

8. Vestbo J, Rennard S. Chronic obstructive pulmonary disease biomarker(s) for disease activity needed-urgently. Am J Respir Crit Care Med 2010;182:863-4.

9. Clement A, Tamalet A, Leroux E, et al. Long term effects of azithromycin in patients with cystic fibrosis: a double blind, placebo controlled trial. Thorax 2006;61:895-902.

10. Wong $\mathrm{C}$, Jayaram L, Karalus $\mathrm{N}$, et al. Azithromycin for prevention of exacerbations in non-cystic fibrosis bronchiectasis (EMBRACE): a randomised, double-blind, placebo-controlled trial. Lancet 2012;380:660-7.

11. Martinez FJ, Curtis JL, Albert R. Role of macrolide therapy in chronic obstructive pulmonary disease. Int J Chron Obstruct Pulmon Dis 2008:3:331-50.

12. Albert RK, Connett J, Bailey WC, et al. Azithromycin for prevention of exacerbations of COPD. N Engl J Med 2011;365:689-98.

13. Verleden SE, Vandooren J, Vos R, et al. Azithromycin decreases MMP-9 expression in the airways of lung transplant recipients. Transpl Immunol 2011;25:159-62.

14. Simpson JL, Powell H, Boyle MJ, et al. Clarithromycin targets neutrophilic airway inflammation in refractory asthma. Am J Respir Crit Care Med 2008:177:148-55.

15. Braber S, Koelink PJ, Henricks PA, et al. Cigarette smoke-induced lung emphysema in mice is associated with prolyl endopeptidase, an enzyme involved in collagen breakdown. Am J Physiol Lung Cell Mol Physiol 2011;300:L255-65.

16. van Houwelingen $\mathrm{AH}$, Weathington NM, Verweij $\mathrm{V}$, et al. Induction of lung emphysema is prevented by L-arginine-threonine-arginine. FASEB J 2008;22:3403-8.

17. Seemungal TA, Donaldson GC, Bhowmik A, et al. Time course and recovery of exacerbations in patients with chronic obstructive pulmonary disease. Am J Respir Crit Care Med 2000;161:1608-13.

18. Woodruff PG. Novel outcomes and end points: biomarkers in chronic obstructive pulmonary disease clinical trials. Proc Am Thorac Soc 2011;8:350-5

19. Papi A, Luppi F, Franco F, et al. Pathophysiology of exacerbations of chronic obstructive pulmonary disease. Proc Am Thorac Soc 2006;3:245-51.

20. Donaldson GC, Seemungal TA, Patel IS, et al. Airway and systemic inflammation and decline in lung function in patients with COPD. Chest 2005;128:1995-2004.

21. Hurst JR, Vestbo J, Anzueto A, et al. Susceptibility to exacerbation in chronic obstructive pulmonary disease. $N$ Engl J Med 2010;363:1128-38.

22. Woodruff PG, Albert R, Chatila W, et al. Analysis of blood biomarkers in the $\mathrm{NIH}$-sponsored azithromycin in COPD Study. Am $J$ Respir Crit Care Med 2012;185:A1007.

23. Wells JM, Jackson PL, O'Reilly PJ, et al. Selective inhibition of leukotriene A4 hydrolase aminopeptidase activity occurs in COPD and reflects clinical outcomes. Am J Respir Crit Care Med 2012;185 A1422. 